\title{
AN ISOMORPHISM AND ISOMETRY THEOREM FOR A CLASS OF LINEAR FUNCTIONALS
}

BY

\author{
WILLIAM D. L. APPLING
}

ABSTRACT. Suppose $U$ is a set, $F$ is a field of subsets of $U$ and $\mathfrak{p}_{A B}$ is the set of all real-valued, finitely additive functions defined on $F$. Two principal notions are considered in this paper. The first of these is that of a subset of $\mathfrak{p}_{A B}$, defined by certain closure properties and called a $C$-set. The second is that of a collection $\mathcal{C}$ of linear transformations from $\mathfrak{p}_{A B}$ into $\mathfrak{p}_{A B}$ with special boundedness properties. Given a $C$-set $M$ which is a linear space, an isometric isomorphism is established from the dual of $M$ onto the set of all elements of $\mathcal{C}$ with range a subset of $M$. As a corollary it is demonstrated that the above-mentioned isomorphism and isometry theorem, together with a previous representation theorem of the author (J. London Math. Soc. 44 (1969), pp. 385-396), imply an analogue of a dual representation theorem of Edwards and Wayment (Trans. Amer. Math. Soc. 154 (1971), pp. 251-265). Finally, a "pseudo-representation theorem" for the dual of $\mathfrak{p}_{A B}$ is demonstrated.

1. Introduction. In a recent paper [5], Edwards and Wayment obtained a set function integral ( $v$-integral) representation theorem for the dual of the space $A C$ of all real-valued functions defined on $[0,1]$ vanishing at 0 and absolutely continuous. In this paper we obtain, among other things, an analogue of the above theorem for finitely additive measure spaces.

Suppose $U$ is a set, $\mathbf{F}$ is a field of subsets of $U, \mathfrak{p}$ is the set of all functions from $\mathbf{F}$ into $R, \mathfrak{p}_{B}$ is the set of all elements of $\mathfrak{p}$ with bounded range, $\mathfrak{p}_{A B}$ is the set of all finitely additive elements of $\mathfrak{p}_{B}$, and $\mathfrak{p}_{A}^{+}$is the set of all nonnegative-valued elements of $\mathfrak{p}_{A B}$. For each $\eta$ in $\mathfrak{p}_{A B}$ and $V$ in $\mathbf{F}, \eta^{|V|}$ denotes the element of $\mathfrak{p}_{A B}$ given by

$$
\eta^{[V]}(I)=\eta(V \cap I) \text {. }
$$

Definition. The statement that $M$ is a $C$-set means that $M \subseteq \mathfrak{p}_{A B}$ such that:

Presented to the Society, November 9, 1972 under the title Concerning duals of linear $C$-sets; received by the editors March 6,1973 and, in revised form, October 2, 1973 and November 19, 1973.

AMS (MOS) subject classifications (1970). Primary 28A25, 28A10; Secondary 47B99.

Key words and phrases. v-integral, isomorphism, isometry, transformation representation. 
(i) if $\eta$ is in $M, \kappa$ is in $\mathfrak{p}_{A B}$ and, for all $V$ in $\mathrm{F}, \int_{V}|\kappa(I)| \leqslant$ $\int_{V}|\eta(I)|$, then $\kappa$ is in $M$, and

(ii) if $\eta$ is in $\mathfrak{p}_{A}^{+}$and $\lambda$ is the function from $\mathbf{F}$ into $\mathbf{R}$ given by $\lambda(V)=\sup \left\{\kappa(I): \kappa\right.$ in $M \cap \mathfrak{p}_{A}^{+}, \eta-\kappa$ in $\left.\mathfrak{p}_{A}^{+}\right\}$, then $\lambda$ is in $M \cap \mathfrak{p}_{A}^{+}$.

The following are examples of $C$-sets:

(1) If $\beta$ is in $\mathfrak{p}_{B}$, then the set of all $\eta$ in $\mathfrak{p}_{A B}$, such that $\int_{U} \beta(I) \eta(I)$ exists, is a $C$-set and a linear space.

(2) If $\beta$ is in $\mathfrak{p}_{B}$, then the set of all $\eta$ in $\mathfrak{p}_{A B}$, such that $\int_{U}|\beta(I)||\eta(I)|$ exists and is 0 , is a $C$-set and a linear space.

(3) If $\kappa$ is in $\mathfrak{p}_{A}^{+}$, then the set of all $\eta$ in $\mathfrak{p}_{A B}$, such that $\kappa-\int|\eta|$ is in $\mathfrak{p}_{A}^{+}$, is a $C$-set.

(4) If $G$ is a collection of $C$-sets, then $\bigcap_{G} X$ is a $C$-set.

(5) If $\mu$ is in $\mathfrak{p}_{A}^{+}$, then the set to which $\eta$ belongs iff $\eta$ is an element of $\mathfrak{p}_{A B}$ such that if $0<c$, then there is a $d>0$ and a subdivision (see $\S 2$ ) $\mathfrak{D}$ of $U$, such that if $I$ is in a refinement (see $\S 2$ ) of $\mathfrak{D}$ and $\mu(I)<d$, then $|\eta(I)|<c$, is a $C$-set and a linear space.

(6) If $\beta$ is in $\mathfrak{p}_{B}$ and $0 \leqslant d$, then the set of all $\eta$ in $\mathfrak{p}_{A B}$, such that the upper and lower integral difference (see [2]) $\int_{U} L(\beta \eta)(I)-G(\beta \eta)(I) \leqslant d$, is a $C$-set.

(7) If $\mu$ is in $\mathfrak{p}_{A}^{+}$, then the set $A_{\mu}$ of all $\eta$ in $\mathfrak{p}_{A B}$ absolutely continuous with respect to $\mu$ is a $C$-set and a linear space.

THEOREM 1.A.1 [4]. Suppose $M$ is a C-set. Suppose that for each $\eta$ in $\mathfrak{p}_{A B}, \tau(\eta)$ and $\lambda(\eta)$ are defined, respectively, as follows:

$$
\tau(\eta)(I)= \begin{cases}1 & \text { if } \eta(I) \geqslant 0 \\ -1 & \text { if } \quad \eta(I)<0\end{cases}
$$

and

$$
\lambda(\eta)(I)=\sup \left\{\kappa(I): \kappa \text { in } M \cap \mathfrak{p}_{A}^{+}, \int|\eta|-\kappa \text { in } \mathfrak{p}_{A}^{+}\right\} .
$$

Then there is a transformation $\mathbf{a}_{M}$ from $\mathfrak{p}_{A B}$ into $M$ such that if $\eta$ is in $\mathfrak{p}_{A B}$ and $V$ is in $\mathbf{F}$, then

$$
\mathbf{a}_{M}(\eta)(V)=\int_{V} \tau(\eta)(I) \lambda(\eta)(I)
$$

furthermore, if $\eta$ is in $\mathfrak{p}_{A B}, \kappa$ is in $M$ and $\kappa \neq \mathrm{a}_{M}(\eta)$, then

$$
\int_{U}\left|\eta(I)-\mathrm{a}_{M}(\eta)(I)<\int_{U}\right| \eta(I)-\kappa(I) \mid .
$$

For each $C$-set $M$, we shall let $\mathrm{a}_{M}$ denote the transformation associated with $M$ by Theorem 1.A.1.

Corollary 1.A.2 [4]. If $M$ is a $C$-set and a linear space, then $a_{M}$ is a linear transformation. 
We let $C$ denote the set to which $T$ belongs iff $T$ is a linear transformation from $\mathfrak{p}_{A B}$ into $\mathfrak{p}_{A B}$ such that, for some $K \geqslant 0$ and all $\eta$ in $\mathfrak{p}_{A B}$ and $V$ in $\mathbf{F}, \int_{V}|T(\eta)(I)| \leqslant K \int_{V}|\eta(I)|$.

The reader can easily see that Theorem 1.A.1 and Corollary 1.A.2 imply the following corollary.

Corollary 1.A.3. If $M$ is a $C$-set and a linear space, then $\mathbf{a}_{M}$ is in C.

The principal theorem of this paper is the following isomorphism and isometry theorem.

TheOREM 4.1. Suppose $M$ is a $C$-set and a linear space. The mapping $\mathrm{X}_{M}$, defined as

$\{(S, E): S$ in the dual of $M, E$ a function with domain $\mathfrak{p}_{A B}$ and range $\subseteq \mathfrak{p}$, given by $E(\eta)(V)=S\left(\mathrm{a}_{M}\left(\eta^{[V]}\right)\right), \eta$ in $\mathfrak{p}_{A B}, V$ in $\left.\mathbf{F}\right\}$

is an isomorphism and an isometry from the dual of $M$ onto the set to which $T$ belongs iff $T$ is in $C$ and the range of $T \subseteq M$, i.e., $\mathrm{a}_{M} T=T$.

THEOREM 1.A.2 [3]. If $T$ is in $\mathrm{C}, \mu$ is in $\mathfrak{p}_{A}^{+}$, and the range of $T$ is a sub. set of $A_{\mu}$, then, for all $\eta$ in $\mathfrak{p}_{A B}$ and $V$ in $\mathbf{F}$,

$$
T(\eta)(V)=\int_{V}[T(\mu)(I) / \mu(I)] \mathbf{a}_{A_{\mu}}(\eta)(I) \quad \text { (v-integral). }
$$

The analogue mentioned above and a consequence of Theorems 4.1 and $1 . A .2$ is as follows $(\S 5)$.

TheOREM 5.1. If $\mu$ is in $\mathfrak{p}_{A}^{+}$, then the function $Y$ defined by

$$
Y=\left\{\left(S,\left\{\left(I, S\left(\mu^{[I]}\right)\right): I \text { in } \mathbf{F}\right\}\right): S \text { in the dual of } A_{\mu}\right\},
$$

is an isomorphism from the dual of $A_{\mu}$ onto $\operatorname{Lip}(\mu)$; furthermore, if $S$ is in the dual of $A_{\mu}$, then

$$
\|S\|=\sup \left\{\left|S\left(\mu^{[I]}\right)\right| / \mu(I): I \text { in } \mathbf{F}\right\},
$$

and for each $\eta$ in $A_{\mu}, S(\eta)=\int_{U}\left[S\left(\mu^{[I]}\right) / \mu(I)\right] \eta(I)$.

For a $\sigma$-algebra $\Sigma$ of subsets of a set $U$, and the Banach space $c a(U, \Sigma)$ of all real-valued countably additive set functions defined on $\Sigma$, with variation norm, Mauldin [7] has obtained, subject to certain cardinality assumptions, a representation for the dual of $c a(U, \Sigma)$. In this paper, for our finitely additive setting, we obtain the following "pseudo-representation theorem" $(\S 6)$.

THEOREM 6.1. If $S$ is a function from $\mathbf{F}$ into $\mathbf{R}$, then the following two statements are equivalent.

(1) $S$ is in the dual of $\mathfrak{p}_{A B}$ (variation norm). 
(2) There is $K \geqslant 0$, a set $W$ with partial ordering $\leqslant *$ with respect to which $W$ is directed, and for each $x$ in $W$, a function $B_{x}$ from $\mathbf{F}$ into $[-K, K]$ such that if $\eta$ is in $\mathfrak{p}_{A B}$, then each of the limits with respect to $\leqslant *$ (see §2),

$$
\lim \int_{U} L\left(\mathrm{~B}_{x} \eta\right)(I) \text { and } \lim \int_{U} G\left(\mathrm{~B}_{x} \eta\right)(I)
$$

exists and is $S(\eta)$.

2. Preliminary theorems and definitions. In the interest of brevity we shall simply refer the reader to [3] for detailed remarks about the notions of subdivision, refinement and integral that we shall use in this paper. We will say here, however, that throughout this paper, as in [3], all integrals are limits for refinements of subdivisions of the appropriate sums. We also refer the reader to [2] for the notions of $\Sigma$-boundedness, sum supremum functional $L$, and sum infimum functional $G$. There are refinement-sum inequalities in [2] that imply the existence of the various integrals that we shall discuss, and it is to [2] that the reader is also referred for a statement of Kolmogoroff's [6] differential equivalence theorem and its implications about the existence and equivalence of various integrals that we will write. Throughout this paper, when the existence of an integral or the equivalence of an integral to an integral is an easy consequence of the above mentioned material, the integral need merely be written or the equivalence assertion made, and the justification left to the reader. We let " $\mathfrak{E} \ll \mathfrak{D}$ " mean "CE is a refinement of $\mathfrak{D}$ ".

3. Some basic properties of $C$ and a commutativity theorem.

Lemma 3.A.1 [3]. If $T$ is in $C$ and $\mu$ is in $\mathfrak{p}_{A}^{+}$, then $T \mathbf{a}_{\mathrm{A}_{\mu}}=\mathrm{a}_{\mathrm{A}_{\mu}} T$. $p_{A}^{+}$.

For the remainder of this paper, we shall let $a_{\mu}$ denote $a_{A_{\mu}}$ for all $\mu$ in

TheOREM 3.A.2 [3]. If $T$ is in $C, S$ is in $C, \mu$ is in $\mathfrak{p}_{A}^{+}$, and the range of $S \subseteq A_{\mu}$, i.e., $\mathrm{a}_{\mu} S=S$, then $T S=S T$.

THEOREM 3.1. If each of $P$ and $Q$ is in $C$, then $P Q=Q P$.

Proof. Suppose $\eta$ is in $\mathfrak{p}_{A B}$. Obviously there is $\kappa$ in $\mathfrak{p}_{A}^{+}$such that $\eta$ is in $A_{\kappa}$, so that $\eta=a_{\kappa}(\eta)$. If $T$ is in C, then, by Lemma 3.A.1,

$$
T \mathrm{a}_{\kappa}=\mathrm{a}_{\kappa} T=\left(\mathrm{a}_{\kappa}\right)^{2} T=\mathrm{a}_{\kappa}\left(\mathrm{a}_{\kappa} T\right)=\mathrm{a}_{\kappa}\left(T \mathrm{a}_{\kappa}\right),
$$

so that if $S$ is in $C$, then, by Theorem 3.A.2, $S\left(T \mathrm{a}_{\kappa}\right)=\left(T \mathrm{a}_{\kappa}\right) S$. This implies that

$$
(P Q) \mathrm{a}_{\kappa}=P\left(Q \mathrm{a}_{\kappa}\right)=\left(Q \mathrm{a}_{\kappa}\right) P=Q\left(\mathrm{a}_{\kappa} P\right)=Q\left(P \mathrm{a}_{\kappa}\right)=(Q P) \mathrm{a}_{\kappa},
$$


so that

$$
\begin{aligned}
(P Q)(\eta) & =(P Q)\left(\mathbf{a}_{\kappa}(\eta)\right)=\left[(P Q) \mathbf{a}_{\kappa}\right](\eta) \\
& =\left[(Q P) \mathbf{a}_{\kappa}\right](\eta)=(Q P)\left(\mathbf{a}_{\kappa}(\eta)\right)=(Q P)(\eta) .
\end{aligned}
$$

Therefore $P Q=Q P$.

4. The isomorphism and isometry theorem. We begin with two lemmas.

LEMmA 4.1. If $T$ is in $C, \eta$ is in $\mathfrak{P}_{A B}$ and $V$ is in $\mathbf{F}$, then

$$
T(\eta)(V)=T\left(\eta^{[V]}\right)(U) \text { and } T\left(\eta^{[V]}\right)=(T(\eta))^{[V]} \text {. }
$$

Proof. There is $K \geqslant 0$ such that $\int_{W}|T(\kappa)(I)| \leqslant K \int_{W}|\kappa(I)|$ for all $\kappa$ in $\mathfrak{P}_{A B}$ and $W$ in $\mathbf{F}$. If $V$ is in $\mathbf{F}$, then

$$
\begin{aligned}
\left|T(\eta)(V)-T\left(\eta^{[V]}\right)(U)\right| & =\left|T(\eta)(V)-T\left(\eta^{[V]}\right)(V)-T\left(\eta^{[V]}\right)(U-V)\right| \\
& =\left|T\left(\eta-\eta^{[V]}\right)(V)-T\left(\eta^{[V]}\right)(U-V)\right| \\
& \leqslant\left|T\left(\eta-\eta^{[V]}\right)(V)\right|+\left|T\left(\eta^{[V]}\right)(U-V)\right| \\
& \leqslant K \int_{V}\left|\eta(I)-\eta^{[V]}(I)\right|+K \int_{U-V}\left|\eta^{[V]}(I)\right| \\
& =K 0+K 0=0 ;
\end{aligned}
$$

so that $T(\eta)(V)=T\left(\eta^{[V]}\right)(U)$.

Now, if each of $W$ and $I$ is in $F$, then from above,

$$
\begin{aligned}
T\left(\eta^{[W]}\right)(I) & =T\left(\left(\eta^{[W]}\right)^{[I]}\right)(U)=T\left(\eta^{[W \cap I]}\right)(U) \\
& =T(\eta)(W \cap I)=(T(\eta))^{[W]}(I) .
\end{aligned}
$$

Therefore, for all $V$ in $\mathrm{F}, T\left(\eta^{[V]}\right)=(T(\eta))^{[V]}$.

LEMmA 4.2. If $T$ is in $C$ and $0 \leqslant H$, then

$$
\int_{U}|T(\eta)(I)| \leqslant H \dot{\int_{U}}|\eta(I)|
$$

for all $\eta$ in $\mathfrak{p}_{A B}$ iff for all $\eta$ in $\mathfrak{p}_{A B}$ and all $V$ in $\mathbf{F}$,

$$
\int_{V}|T(\eta)(I)| \leqslant H \int_{V}|\eta(I)| .
$$

Proof. Obviously the second statement implies the first.

Suppose the first of the statements is true.

If $V$ is in $\mathbf{F}$ and $\mathfrak{D} \ll\{V\}$, then

$$
\begin{aligned}
\sum_{D}|T(\eta)(I)| & =\sum_{D}\left|T\left(\eta^{[I]}\right)(U)\right| \leqslant \sum_{D} H \int_{U}\left|\eta^{[I]}(J)\right| \\
& =\sum_{D} H \int_{I}|\eta(J)|=H \int_{V}|\eta(J)|,
\end{aligned}
$$

so that $\int_{V}|T(\eta)(I)| \leqslant H \int_{V} \ln (J) \mid$. 
We now prove Theorem 4.1, as stated in the introduction.

Proof of Theorem 4.1. Suppose $S$ is in the dual of $M$ and $E$ is the function with domain $\mathfrak{p}_{A B}$ and range $\subseteq \mathfrak{p}$ given by $E(\eta)(V)=S\left(\mathrm{a}_{M}\left(\eta^{[V]}\right)\right)$. It is clear that if $\eta$ is in $\mathfrak{p}_{A B}$, then $E(\eta)$ is finitely additive; furthermore, if $V$ is in $\mathbf{F}$, then

$$
\begin{aligned}
|E(\eta)(V)| & =\left|S\left(\mathbf{a}_{M}\left(\eta^{[V]}\right)\right)\right| \leqslant\|S\| \int_{U}\left|\mathbf{a}_{M}\left(\eta^{[V]}\right)(I)\right| \\
& =\|S\| \int_{V}\left|\mathbf{a}_{M}(\eta)(I)\right| .
\end{aligned}
$$

This implies that $E(\eta)$ is in $\mathfrak{p}_{A B}$ and that if $W$ is in $\mathbf{F}$, then $\int_{W}|E(\eta)(J)| \leqslant$ $\|S\| \int_{W}\left|\mathrm{a}_{M}(\eta)(I)\right|$, so that, from the assumption that $M$ is a linear space as well as a $C$-set, we have that $E(\eta)$ is in $M$. Thus $a_{M} E=E$. It is obvious that $E$ is linear, and from the immediately preceding inequality, Lemma 4.2 and the fact that $\left\|\mathrm{a}_{M}\right\| \leqslant 1$, it follows that $E$ is in $C$ and $\|E\| \leqslant\|S\|$. Now, if $\kappa$ is in $M$, then

$$
\begin{aligned}
|S(\kappa)| & =\left|S\left(\mathrm{a}_{M}\left({ }^{[U]}\right)\right)\right|=|E(\kappa)(U)| \\
& \leqslant \int_{U}|E(\kappa)(I)| \leqslant\|E\| \int_{U}|\kappa(I)| .
\end{aligned}
$$

Therefore $\|S\| \leqslant\|E\|$. Therefore $\|S\|=\|E\|$.

It is an easy consequence of the definition of $\mathrm{X}_{M}$ that $\mathrm{X}_{M}$ is linear, so that we therefore finally have that $\mathrm{X}_{M}$ is an "isomorphic isometry from the dual of $M$ 'into' $\left\{T: T\right.$ in $\left.C, \mathrm{a}_{M} T=T\right\}$." We now show that $\mathrm{X}_{M}$ is "onto". Suppose $T$ is in $C$ and $\mathrm{a}_{M} T=T$. Let $Q$ be the function from $M$ into $\mathbf{R}$ given by $Q(\kappa)=T(\kappa)(U)$. Obviously $Q$ is in the dual of $M$. Now, if $\eta$ is in $\mathfrak{p}_{A B}$ and $V$ is in $F$, then

$$
\begin{aligned}
\mathrm{X}_{M}(Q)(\eta)(V) & =Q\left(\mathrm{a}_{M}\left(\eta^{[V]}\right)\right)=T\left(\mathrm{a}_{M}\left(\eta^{[V]}\right)\right)(U)=T\left(\left[\mathrm{a}_{M}(\eta)\right]^{[V]}\right)(U) \\
& =T\left(\mathrm{a}_{M}(\eta)\right)(V)=\mathrm{a}_{M}(T(\eta))(V)=T(\eta)(V),
\end{aligned}
$$

so that $\mathrm{X}_{M}(Q)=T$ and therefore $\mathrm{X}_{M}$ is "onto".

5. The dual of $A_{\mu}$. In this section we prove Theorem 5.1, as stated in the introduction.

We begin by stating a previous theorem of the author [1].

THEOREM 5.A.1. If $\alpha$ is in $\mathfrak{p}_{B}, \mu$ is in $\mathfrak{p}_{A}^{+}, \eta$ is in $A_{\mu} \cap \mathfrak{p}_{A}^{+}$and $\int_{U} \alpha(I) \mu(I)$ exists, then $\int_{U} \alpha(I) \eta(I)$ exists.

Corollary 5.A1. If $\alpha$ is in $\mathfrak{p}_{B}, \mu$ is in $\mathfrak{p}_{A}^{+}, \kappa$ is in $A_{\mu}$ and $\int_{U} \alpha(I) \mu(I)$ exists, then $\int_{U} \alpha(I) \kappa(I)$ exists.

Proof. Let $\eta=\int|\kappa| . \quad \eta$ is in $A_{\mu} \cap p_{A}^{+}$. By Theorem 5.A.1, $\int_{U} \alpha(I) \eta(I)$ exists. Since $\kappa / \eta$ is in $p_{B}$ and $\kappa=\int[\kappa / \eta] \eta$, it follows (see $\S 2$ ) 
that $\int_{U} \alpha(I)[\kappa(I) / \eta(I)] \eta(I)$ exists. But clearly $\int_{U} \alpha(I)[\kappa(I) / \eta(I)] \eta(I)=$ $\int_{U} \alpha(I) \kappa(I)$.

Proof of Theorem 5.1. It is clear that if $S$ is in the dual of $A_{\mu}$, then $Y(S)$ is in $\operatorname{Lip}(\mu)$, and that $Y$ preserves addition and scalar multiplication. Let $M=A_{\mu}$. Then $\mathrm{a}_{M}=\mathrm{a}_{\mu}$, as defined in Theorem 1.A.1. Suppose $S$ is in the dual of $M$ and let $T=\mathrm{X}_{M}(S)$. If $\eta$ is in $A_{\mu}$ and $I$ is in $F$, then

$$
T(\eta)(I)=S\left(\mathrm{a}_{\mu}\left(\eta^{[I]}\right)\right)=S\left(\eta^{[I]}\right) .
$$

Therefore if $\kappa$ is in $A_{\mu}$, then, by definition of $X_{M}$ and Theorem 1.A.2,

$$
\begin{aligned}
S(\kappa) & =S\left(\kappa^{[U]}\right)=T(\kappa)(U)=\int_{U}[T(\mu)(I) / \mu(I)] \mathrm{a}_{\mu}(\kappa)(I) \\
& =\int_{U}\left[S\left(\mu^{[I]}\right) / \mu(I)\right] \kappa(I) .
\end{aligned}
$$

Let $N=\sup \left\{\left|S\left(\mu^{[I]}\right)\right| / \mu(I): I\right.$ in $\left.\mathbf{F}\right\}$. If $\kappa$ is in $A_{\mu}$, then

$$
\begin{aligned}
|S(\kappa)| & =\left|\int_{U}\left[S\left(\mu^{[I]}\right) / \mu(I)\right] \kappa(I)\right| \\
& \leqslant \int_{U}\left[\left|S\left(\mu^{[I]}\right)\right| / \mu(I)\right]|\kappa(I)| \leqslant N \int_{U}|\kappa(I)| .
\end{aligned}
$$

Therefore $\|S\| \leqslant N$. Now, if $I$ is in $\mathbf{F}$, then

$$
\|S\| \geqslant\left|S\left(\mu^{[I]}\right)\right| / \mu^{[I]}(U)=\left|S\left(\mu^{[I]}\right)\right| / \mu(I) .
$$

Therefore $N \leqslant\|S\|$. Therefore $\left.\|S\|=\sup \left\{\left|S\left(\mu^{[I]}\right)\right| / \mu\right) I\right): I$ in $\left.\mathbf{F}\right\}$.

We see that the fact that $Y$ is invertible follows readily from the above integral representation for the elements of the dual of $A_{\mu}$.

We now show that $Y$ is "onto". Suppose $\kappa$ is in $\operatorname{Lip}(\mu)$. The function $\kappa / \mu$ is in $\mathfrak{p}_{B}$ and it is obvious that $\kappa=\int[\kappa / \mu] \mu$. From Corollary 5.A.1 it follows that if $\eta$ is in $A_{\mu}$, then $\int_{U}[\kappa(I) / \mu(I)] \eta(I)$ exists, and we clearly see that $T$, defined by

$$
T=\left\{\left(\iota, \int_{U}[\kappa(I) / \mu(I)] \iota(I)\right): \iota \text { in } A_{\mu}\right\}
$$

is in the dual of $A_{\mu}$. Furthermore, if $V$ is in $F$, then

$$
\begin{aligned}
Y(T)(V) & =T\left(\mu^{[V]}\right)=\int_{U}[\kappa(I) / \mu(I)] \mu^{[V]}(I) \\
& =\int_{V}[\kappa(I) / \mu(I)] \mu(I)=\kappa(V) .
\end{aligned}
$$

Thus $Y(T)=\kappa$.

6. The dual of $\mathfrak{p}_{A B}$. Let us begin by noting, trivially, that $\mathfrak{p}_{A B}$ is a $C$. set and a linear space, and that therefore $\mathrm{X}_{\mathrm{p}_{A B}}$ is an isomorphism and an isometry from the dual of $\mathfrak{p}_{A B}$ onto $C$. Furthermore, $a_{\mathfrak{p}_{A B}}$ is clearly the identity transformation, so that if $S$ is in the dual of $\mathfrak{p}_{A B}, \eta$ is in $\mathfrak{p}_{A B}$ and $V$ is in 
F, then

$$
X_{\mathfrak{p}_{A B}}(S)(\eta)(V)=S\left(\mathrm{a}_{\mathfrak{p}_{A B}}\left(\eta^{[V]}\right)\right)=S\left(\eta^{[V]}\right) .
$$

We now prove Theorem 6.1, as stated in the introduction.

Proof of Theorem 6.1. Suppose (2) is true. If $x$ is in $W, \eta$ is in $\mathfrak{p}_{A B}$, $V$ is in $\mathbf{F}$ and $\mathfrak{D} \ll\{V\}$, and for each $I$ in $\mathfrak{D}, \beta_{x}(I)$ is in $\mathbf{B}_{x}(I)$, then

$$
\left|\sum_{\mathcal{D}} b_{x}(I) \eta(I)\right| \leqslant \sum_{\mathcal{D}}\left|b_{x}(I)\right||\eta(I)| \leqslant K \int_{V}|\eta(I)|,
$$

so that if $Q$ is $L$ or $G$, then $\left|Q\left(\mathrm{~B}_{x} \eta\right)(V)\right| \leqslant K \int_{V}|\eta(I)|$, which implies that

$$
\left|\int_{U} Q\left(\mathrm{~B}_{x} \eta\right)(V)\right| \leqslant \int_{U}\left|Q\left(\mathrm{~B}_{x} \eta\right)(V)\right| \leqslant K \int_{U}|\eta(V)|,
$$

so that $|S(\eta)| \leqslant K \int_{U}|\eta(V)|$.

We now show that $S$ is linear. Suppose each of $r$ and $s$ is in $\mathbf{R}$ and each of $\eta$ and $\kappa$ is in $\mathfrak{p}_{A B}$. Suppose $0<c$. From the fact that $W$ is directed with respect to $\leqslant^{*}$, it follows that there is $\mathrm{X}$ in $W$ such that if $y$ is in $W$ and $\mathrm{X} \leqslant * y$ and $Q$ is $L$ or $G$ and $\rho$ is either $\eta, \kappa, r \eta, s \kappa$, or $r \eta+s \kappa$, then

$$
\left|S(\rho)-\int_{U} Q\left(\mathrm{~B}_{y} \rho\right)(I)\right|<c /[8(1+|r|+|s|)] .
$$

There is $\mathfrak{D} \ll\{U\}$ such that if $\mathfrak{C} \ll \mathfrak{D}$ and $Q$ and $\rho$ are as above, then

$$
\left|\int_{U} Q\left(\mathrm{~B}_{X} \rho\right)(I)-\sum_{C} Q\left(\mathrm{~B}_{X} \cdot \rho\right)(I)\right|<c /[8(1+|r|+|s|)],
$$

so that

$$
\left|S(\rho)-\sum_{G} Q\left(\mathrm{~B}_{X} \rho\right)(I)\right|<c /[4(1+|r|+|s|)] .
$$

Now, if $\rho$, once again, is as above, and for each $I$ in $\mathfrak{D}, b_{X}(I)$ is in $\mathrm{B}_{X}(I)$, then

$$
\sum_{D} G\left(\mathrm{~B}_{X} \rho\right)(I)^{\varphi} \leqslant \sum_{\mathcal{D}} b_{X}(I) \rho(I) \leqslant \sum_{\mathcal{D}} L\left(\mathrm{~B}_{X} \rho\right)(I)
$$

so that

$$
\left|S(\rho)-\sum_{D} b_{X}(I) \rho(I)\right|<c /[4(1+|r|+|s|)] .
$$


Therefore

$$
\begin{aligned}
& |S(r \eta+s \kappa)-[r S(\eta)+s S(\kappa)]|=\mid S(r \eta+s \kappa)-\sum_{D} b_{X}(I)[r \eta(I)+s \kappa(I)] \\
& +r\left\{\sum_{D} b_{X}(I) \eta(I)-S(\eta)\right\} \\
& +s\left\{\sum_{D} b_{X}(I) \kappa(I)-S(\kappa)\right\} \mid \\
& \leqslant\left|s(r \eta+s k)-\sum_{D} b_{X}(I)[r \eta(I)+s k(I)]\right| \\
& +|r|\left|\sum_{D} b_{X}(I) \eta(I)-S(\eta)\right| \\
& +|s|\left|\sum_{D} b_{X}(I) \kappa(I)-S(\kappa)\right| \\
& <c /[4(1+|r|+|s|)] \\
& +|r| c /[4(1+|r|+|s|)] \\
& +|s| c /[4(1+|r|+|s|)] \\
& <3 c / 4<c \text {. }
\end{aligned}
$$

Therefore $S(r \eta+s \kappa)=r S(\eta)+s S(\kappa)$. Therefore $S$ is in the dual of $p_{A B}$. Therefore (2) implies (1).

Now suppose (1) is true, i.e., $S$ is in the dual of $\mathfrak{p}_{A B}$. Let $T=\mathrm{X}_{\mathfrak{p}_{A B}}(S)$, so that, with reference to the opening paragraph of this section, $T$ is the element of $\mathcal{C}$ given by $T(\eta)(V)=S\left(\eta^{[V]}\right)$.

We seek a set, $W$, a partial ordering, $\leqslant^{*}$, for $W$ with respect to which $W$ is directed, a number $K \geqslant 0$, and for each $y$ in $W$, a function $\mathbf{B}_{y}$ from $\mathbf{F}$ into $[-K, K]$ such that if $\eta$ is in $A B$ and $Q$ is $L$ or $G$, then $\int_{U} Q\left(\mathrm{~B}_{y} \eta\right)(I) \rightarrow S(\eta)$, limit for $\leqslant^{*}$. Let $W=\mathfrak{p}_{A}^{+}$, and let $\leqslant *=\{(\eta, \kappa): \eta, \kappa$ and $\kappa-\eta$ in $\mathfrak{p}_{A}^{+}$\}. It is clear that $\leqslant^{*}$ is a partial ordering, and from the fact that if $V$ is in $\mathbf{F}$ and each of $\rho$ and $\nu$ is in $\mathfrak{p}_{A}^{+}$, then $\int_{V} \max \{\rho(I), \eta(I)\}$ exists, it follows that $\mathfrak{p}_{A}^{+}$is directed with respect to $\leqslant *$. For each $v$ in $\mathfrak{p}_{A}^{+}$ and $V$ in $\mathrm{F}$, let

$$
\beta_{v}(V)=T(v)(V) / v(V) .
$$

Letting $K=\|S\|$, we see that for each $v$ in $\mathfrak{p}_{A}^{+}, \mathrm{B}_{v}$ is a function from $\mathbf{F}$ into $[-K, K]$. Only the final limiting assertion remains to be shown. Suppose $\eta$ is in $\mathfrak{p}_{A B}$. Let $\mu$ be the element of $\mathfrak{p}_{A}^{+}$given by $\mu(V)=\int_{V}|\eta(I)|$. If $v$ is in $\mathfrak{p}_{A}^{+}$and $\mu \leqslant^{*} v$, then $\eta$ is in $A_{v}$, so that by Theorem 1.A.4, stated in the introduction, for each $V$ in $F$,

$$
T(\eta)(V)=\int_{V}[T(v)(I) / v(I)] \eta(I),
$$


so that if $Q$ is $L$ or $G$, then

$$
\begin{aligned}
S\left(\eta^{[V]}\right) & =T(\eta)(V)=\int_{V}[T(v)(I) / v(I)] \eta(I) \\
& =\int_{V} \mathrm{~B}_{v}(I) \eta(I)=\int_{V} Q\left(\mathrm{~B}_{v} \eta\right)(I) .
\end{aligned}
$$

This implies $\int_{V} Q\left(\mathrm{~B}_{v} \eta\right)(I) \rightarrow S\left(\eta^{[V]}\right)$ "uniformly in F" with respect to $\leqslant^{*}$; in particular,

$$
\int_{U} Q\left(\mathrm{~B}_{v} \eta\right)(I) \rightarrow S\left(\eta^{[U]}\right)=S(\eta)
$$

with respect to $\leqslant *$. Therefore (1) implies (2). Therefore (1) and (2) are equivalent.

\section{REFERENCES}

1. W. D. L. Appling, Some integral characterizations of absolute continuity, Proc. Amer. Math. Soc. 18 (1967), 94-99. MR 34 \#4446.

2. - Summability of real-valued set functions, Riv. Mat. Univ. Parma (2) 8 (1967), 77-100. MR 40 \#4418.

3. - Concerning a class of linear transformations, J. London Math. Soc. 44 (1969), 385-396. MR 38 \#6015.

4. - A generalization of absolute continuity and of an analogue of the Lebesgue decomposition theorem, Riv. Mat. Univ. Parma (to appear).

5. J. R. Edwards and S. G. Wayment, Representations for transformations continuous in the BV norm, Trans. Amer. Math. Soc. 154 (1971), 251-265. MR 43 \#466.

6. A. Kolmogoroff, Untersuchen über den Integralbegriff, Math. Ann. 103 (1930), 654-696.

7. D. Mauldin, An integral representation of functionals on $c a(S, \Sigma)$, preliminary report, Notices Amer. Math. Soc. 18 (1971), 949. Abstract \#71 T-B198.

DEPARTMENT OF MATHEMATICS, NORTH TEXAS STATE UNIVERSITY, DENTON, TEXAS 76203 